\title{
THE SPECTRUM OF AN OPERATOR IN BANACH SPACE
}

\author{
M. D. GEORGE ${ }^{1}$
}

1. It is well known that if $T$ is a bounded linear transformation in a Hilbert space $X$, then the spectrum of $T$ is contained in the closure of the range of the functional $(T x, x)$ as $x$ varies over the unit sphere of $X$. In [2], Lumer obtained a partial generalization of this result for an arbitrary Banach space by endowing the space with a semiinner-product. In this note, a complete generalization is obtained for a certain class of Banach spaces, using the work of James [1] on orthogonality in normed linear spaces.

2. In what follows, $X$ denotes a Banach space over the complex field $C$ having the following two properties:

(i) $X$ is reflexive;

(ii) the norm in $X$ has a Gateaux differential at each nonzero point, i.e.,

$$
G(x ; y)=\lim _{h \rightarrow 0} \frac{1}{h}\{\|x+h y\|-\|x\|\}
$$

exists for every $x \neq 0$ and $y$ in $X$ (here $h$ is real).

Lemma 1. For any $x \neq 0, y, z$ in $X$ and $t$ real:

(a) $G(x ; x)=\|x\|, G(x ; i x)=0$,

(b) $G(x ; y+z)=G(x ; y)+G(x ; z)$,

(c) $G(x ; t y)=t G(x ; y)$.

The proofs of these are entirely elementary and are omitted.

We will need later some theorems due to James [1] concerning orthogonality in normed linear spaces as defined by Birkhoff. Although his work was done in real spaces, those of his results we need can be carried over to the complex case rather easily. These are listed below, the necessary modifications of James' proofs being indicated where they are not obvious.

Definition. $x$ is orthogonal to $y(x \perp y)$ if for all $k$ in $C,\|x+k y\|$ $\geqq\|x\|$.

Theorem 1 [1, Corollary 2.2, Theorem 4.1]. Suppose $x, y \in X$, $x \neq 0$. Then there exists a unique $a(x, y) \in C$ such that $x \perp a x+y$. Furthermore $|a| \leqq\|y\| /\|x\|$.

Received by the editors August 10, 1964.

1 This research was supported by the Air Force Office of Scientific Research. 
Proof. The existence of $a$ and the estimate for $a$ follow exactly as in James. For the uniqueness, note that if $x \perp a x+y, x \neq 0$, then $G(x ; a x+y)=G(x ; i a x+i y)=0$. Writing $a=\alpha+i \beta$ and using Lemma 1 , it follows that $a$ is uniquely determined, namely

$$
a(x, y)=\frac{1}{\|x\|}\{-G(x ; y)+i G(x ; i y)\}
$$

Theorem 2 [1, Theorem 4.2]. If $x \perp y, x \perp z$, then $x \perp y+z$.

If we extend the definition of $a(x, y)$ to all pairs in $X \times X$ by setting $a(0, y)=0$ for all $y \in X$, then it is routine to check, using Lemma 1 , that $[x, y]=-\|y\|^{2} a(y, x)$ is a semi-inner-product on $X$, as defined in [2]. Indeed, since the unit sphere in $X$ is smooth by virtue of (ii) above, this is the only semi-inner-product on $X$ which agrees with the norm. From Theorem 4 of [2], we now know that the approximate point spectrum of a bounded linear transformation $T$ on $X$ is contained in the closure of the range of the functional $-a(x, T x)$ as $x$ varies over $\{x \mid\|x\|=1\}$. In fact, as we show in the next section, the entire spectrum of $T$ has this property.

3. The next theorem is stated in somewhat more generality than we need; the present form, however, may have some interest in its own right in perturbation problems.

THEOREM 3. Let $L$ be a linear transformation, not necessarily bounded, with domain $D(L)$ and range $R(L)$ dense in $X$. Suppose $L$ has a continuous inverse defined on $R(L)$, whose extension to a bounded operator on $X$ we continue to denote by $L^{-1}$. Let $T$ be a bounded linear transformation $X \in X$ and suppose that for each $x \in X$ with $\|x\|=1, a\left(x, T L^{-1} x\right)$ $\neq 1$. Then $R(L+T)$ is dense in $X$.

Proof. Suppose $\mathrm{Cl}(R(L+T))$ is a proper closed linear subspace of $X$. Using (i), we can apply Theorem 7.3 of [1] (which generalizes easily to the complex case) to conclude that there is an $x \neq 0$ orthogonal to $\mathrm{Cl}(R(L+T))$. By the homogeneity of orthogonality, we may assume $\|x\|=1$. There is a sequence $\left\{w_{n}\right\}, w_{n} \in D(L)$, with $L w_{n} \rightarrow x$. Now

$$
x \perp(L+T) w_{n}=L w_{n}+T L^{-1} L w_{n}
$$

and

$$
x \perp a\left(x, T L^{-1} x\right) x+T L^{-1} x .
$$

Using Theorem 2 and homogeneity, we conclude 


$$
\begin{array}{r}
x \perp L w_{n}+T L^{-1} L w_{n}-a\left(x, T L^{-1} x\right) x-T L^{-1} x \\
=L w_{n}-a\left(x, T L^{-1} x\right) x+T L^{-1}\left(L w_{n}-x\right) .
\end{array}
$$

Passing to the limit as $n \rightarrow \infty$, it is easy to check that

$$
x \perp\left(1-a\left(x, T L^{-1} x\right)\right) x=A x .
$$

Since $A \neq 0$, it follows from the definition of orthogonality that $x=0$. This contradiction completes the proof.

Notice that no restriction is placed on $\|T\|$ in this theorem. Now the main result follows quite easily.

Definition. For $T$ a bounded linear transformation $X \rightarrow X, W(T)$ is the range of the functional $-a(x, T x)$ for $\|x\|=1$.

TheOREM 4. Let $T$ be a bounded linear transformation $X \rightarrow X$, with spectrum $\sigma(T)$. Then $\sigma(T) \subset \mathrm{Cl}(W(T))$.

Proof. Suppose $\lambda \notin \mathrm{Cl}(W(T))$. Then $a(x, T x)$ is never $-\lambda$ for $\|x\|=1$, and $a(x,(\lambda-1) x-T x)$ is never 1 for $\|x\|=1$. Writing $\lambda I-T$ $=I+(\lambda-1) I-T$, it now follows from Theorem 3 that $R(\lambda I-T)$ is dense in $X$. From Theorem 1 ,

$$
\begin{aligned}
\inf _{\|x\|=1}\|(\lambda I-T) x\| & \geqq \inf _{\|x\|=1}|a(x,(\lambda I-T) x)| \\
& =\inf _{\|x\|=1}|\lambda+a(x, T x)|>0 .
\end{aligned}
$$

Hence, $\lambda I-T$ has a continuous inverse, so $\lambda \notin \sigma(T)$ and the proof is complete.

\section{REFERENCES}

1. R. C. James, Orthogonality and linear functionals in normed linear spaces, Trans. Amer. Math. Soc. 61 (1947), 265-292.

2. G. Lumer, Semi-inner-product spaces, Trans. Amer. Math. Soc. 100 (1961), $29-43$.

UNIVERSITY OF MISSOURI 